\title{
Demonstration of Single-Mode Multicore Fiber Transport Network with Crosstalk-Aware In-Service Optical Path Control
}

\author{
Takafumi Tanaka, Klaus Pulverer, Ulrich Häbel, Carlos Castro, Marc Bohn, \\ Takayuki Mizuno, Member, IEEE, Akira Isoda, Kohki Shibahara, Member, IEEE, Member, OSA, \\ Tetsuro Inui, Member, IEEE, Yutaka Miyamoto, Member, IEEE, Yusuke Sasaki, Yoshimichi Amma, \\ Kazuhiko Aikawa, Saurabh Jain, Member, IEEE, Member, OSA, Yongmin Jung, Member, IEEE, \\ Shaif-ul Alam, Senior Member, IEEE, Member, OSA, David J. Richardson, Fellow, IEEE, Fellow, OSA, \\ Md. Nooruzzaman, Member, IEEE, and Toshio Morioka, Member, IEEE, Fellow, OSA
}

\begin{abstract}
Multicore fiber (MCF) transmission is considered as one of the promising technologies for breaking the capacity limit of traditional single mode fibers (SMFs). In addition to achieving longer-distance and larger-capacity transmission, managing inter-core crosstalk (XT) and configuring optical path adaptively based on the XT are important factors in MCF transmission in metro/core backbone networks feasible. In real MCF network, the inter-core XT in a particular core is likely to change continously as the optical paths in the adjacent cores are dynamically assigned to match the dynamic nature of the data traffic. Therefore, monitoring the inter-core $X T$ value continuously and configuring optical path parameters adaptively and flexibly are essential. To address these challenges, we develop an MCF transport network testbed and demonstrate an XT-aware traffic engineering scenario. With the help of a software-defined network (SDN) controller, the modulation format and optical path route are adaptively changed based on the monitored XT values by using programmable devices such as real-time transponder and reconfigurable optical add-drop multiplexer (ROADM).
\end{abstract}

Index Terms-Coherent communications, crosstalk, multicore fiber (MCF), optical communication systems, optical fiber communication, space division multiplexing (SDM), software defined networking (SDN).

\section{INTRODUCTION}

$\mathrm{I}_{\mathrm{on}}$ $\mathrm{N}$ recent years, space-division multiplexing (SDM) in optical fiber transmission has become one of the hottest approaches to break the capacity limit of single-mode fiber (SMF) in fiber optic communication systems [1,2]. Besides the attempts to demonstrate larger capacities and longer transmission distances, various network testbed experiments

T. Tanaka, T. Mizuno, A. Isoda, K. Shibahara, T. Inui, and Y. Miyamoto are with the NTT Network Innovation Laboratories, NTT Corporation, Yokosuka 239-0847, Japan (email: tanaka.takafumi@lab.ntt.co.jp).

K. Pulverer, U. Häbel, C. Castro, and M. Bohn are with the Coriant R\&D GmbH, Munich, 81541, Germany.

Y. Sasaki, Y. Amma, and K. Aikawa are with the Fujikura Ltd., Sakura 285-8550, Japan.

S. Jain, Y. Jung, S. Alam, and D. J. Richardson are with the Optelectronics Research Centre, University of Southampton, Southampton SO17 1BJ, U.K.

M. Nooruzzaman and T. Morioka are with the DTU Fotonik, Technical University of Denmark, Kgs. Lyngby DK2800, Denmark.
[3-5] and optical path provisioning schemes for MCF-based transport networks [6] have been reported. Among the SDM variants, single-mode MCF transport offers highly unique advantages and possibilities. For example, Pbit/s/fiber-class capacities and low-power optical transport networks are feasible by using dense-SDM (DSDM) with spatial multiplicity over 30 [7-9]. In addition, the long-haul digital coherent transmission employed over conventional SMF today can fully be supported by using appropriate crosstalk (XT) management [10]. However, no testbed demonstrations have been reported so far that simultaneously realize these two advantages.

In this paper, we elaborate the first single-mode $\mathrm{MCF}$ transport network using XT-aware and programmable optical paths with XT monitoring [11]. We use the testbed to demonstrate a traffic engineering use case, in which optical paths are adaptively (re)configured subject to consideration of inter-core XT with the help of an SDN controller.

This paper is organized as follows. Section II presents the MCF transport network architecture and XT-aware optical path control scheme applied in the network. Section III explains the testbed setup, which includes a testbed scenario, a MCF transmission line structure, and a SDN controller system architecture. Section IV described the experimental setup and results, and Section $\mathrm{V}$ summarizes the main contents and concludes the paper.

\section{MCF TRANSPORT NETWORK ARCHITECTURE WITH XT-AWARE OPTICAL PATH CONTROL SCHEME}

\section{A. MCF Transport Network Architecture}

As transmission distances increase in MCF transport networks, inter-core XT becomes a limiting factor - especially in the optical paths using higher order modulation formats and high-core-count MCF systems [12]. In addition, the XT impairment will change over time in response to changes in optical path assignments to the cores. Therefore, careful consideration of XT in the phase of optical path provisioning or reconfiguration is indispensable to realize the full potential of MCF transport networks. 


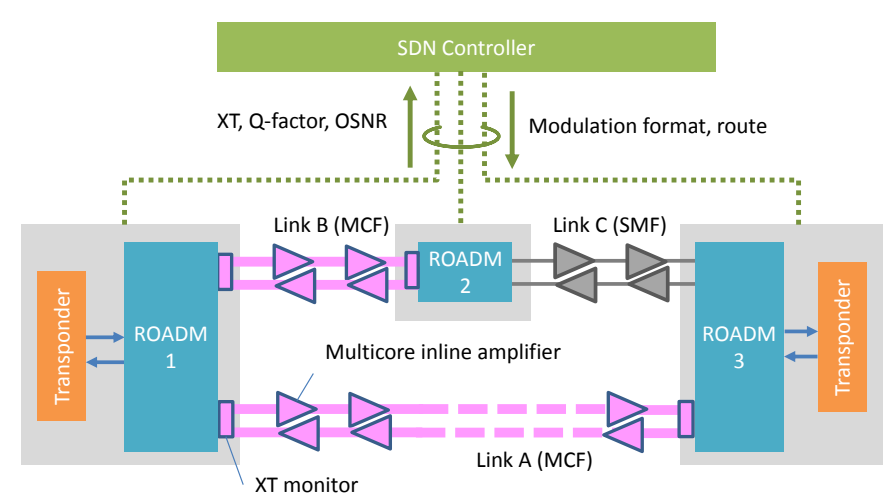

Fig. 1. XT-aware MCF transport network architecture with SDN controller.

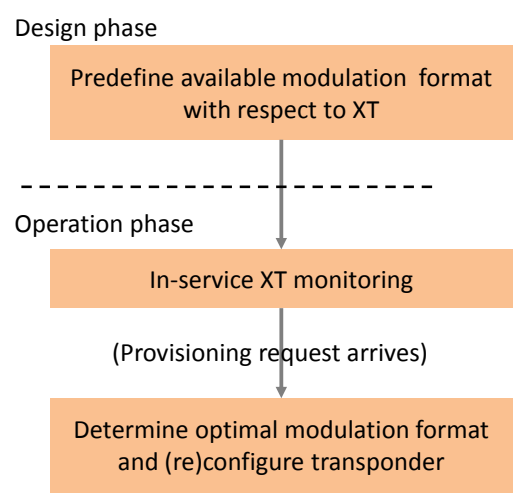

Fig. 2. Flow chart of XT-aware optical channel control scheme.

Figure 1 shows the proposed XT-aware single-mode MCF transport network architecture. This network assumes that MCFs and SMFs are mixed in the process of replacing SMFs with MCFs. In the data-plane, three ROADMs are connected to three SMF/MCF links which include inline amplifiers. In particular, the MCF links contain fan-in/fan-out (FI/FO) devices, and inter-core XT monitors to estimate the XT value within the link. The transponders have the ability to adaptively select the modulation formats of quadrature phase shift keying (QPSK), 8 quadrature amplitude modulation (8QAM), and 16QAM. An optical path between ROADM 1 and 3 traverses either a MCF link (Link A) or mixed MCF and SMF links (Link B-C). In the control plane, the SDN controller manages resource abstraction within the transport network context and provides the interface for northbound service management. The SDN controller collects transmission performance data such as inter-core XT, Q-factor, and optical signal-to-noise ratio (OSNR) values at regular intervals from each node. Based on the monitored values, the SDN controller sets the transponders to an appropriate modulation format and/or configures the ROADMs to change optical path routes.

\section{B. XT-aware Optical Path Control Scheme}

An XT-aware optical path provisioning scheme consists of three steps; one is in the design phase while the other two are in the operational phase (Fig. 2).

In the first step, we predefine the available modulation formats with respect to XT. In SMF-based transport networks, we determine the optimal modulation format from just the

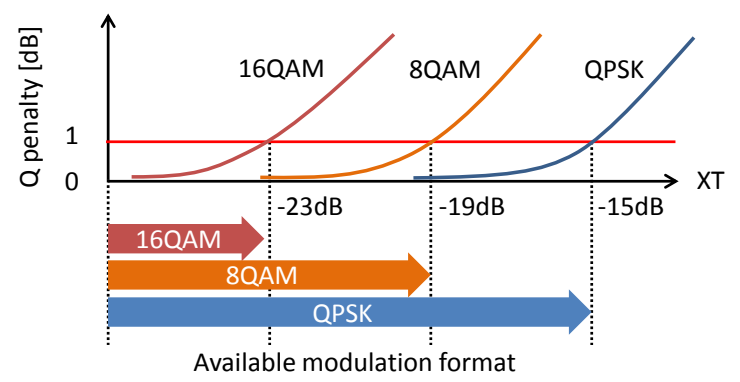

Fig. 3. Relationship between Q penalty and XT, and available modulation formats with respect to XT

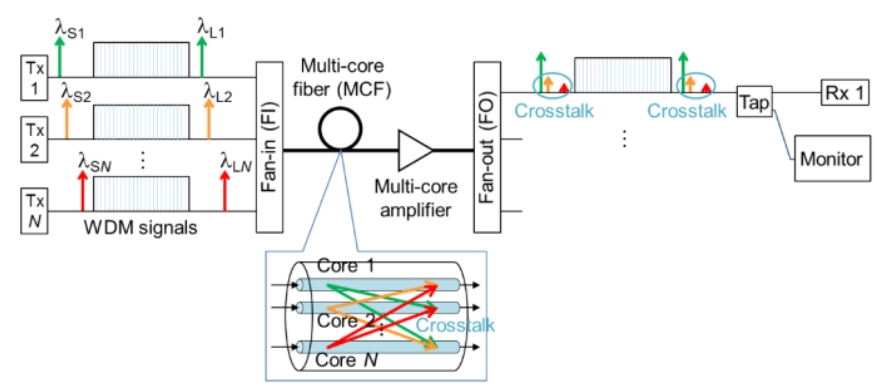

Fig. 4. In-service inter-core XT monitoring scheme [10].

OSNR of the total link. In MCF-based transport networks, the selection of the format depends not only on the OSNR but also on the XT induced in the MCF link. Figure 3 presents the conceptual diagram of available modulation formats with respect to XT. Since the relationship between $\mathrm{Q}$ penalty and XT is basically fully determined by the modulation format, it can be applied to any length of MCF links in the transport network. The allowable Q penalty due to XT should be kept at a sufficiently low level (typically $<1 \mathrm{~dB}$ as shown in Fig. 3) because XT impairment is likely to cause burst errors that degrade the performance of forward error correction (FEC) code. If the allowable $\mathrm{Q}$ penalty due to XT is chosen to be $<1$ $\mathrm{dB}$, the XT requirements for 16QAM-, 8QAM-, and QPSK-modulated signals are $<-23 \mathrm{~dB},<-19 \mathrm{~dB}$, and $<-15$ $\mathrm{dB}$, respectively.

In the second step, the XT value is monitored during operation. We need to monitor the value continuously or periodically in the operation phase because XT impairment may change dynamically over time as optical path assignment conditions in adjacent cores are likely to be modified. In addition, "in-service" XT measurement, which means that the XT monitoring method should not affect or interrupt the WDM signals, is also important for ensuring high-quality network services. This work adopts the in-service XT measurement scheme proposed and demonstrated in [10]. As illustrated in Fig. 4, the scheme adds distinct pilot tones to all cores, one in the shorter and one in the longer wavelength bandwidth used for the wavelength division multiplexed (WDM) signals, so that the XT transfer function can be estimated from the difference between the power of the pilot tone of the core under test and that of other cores. We implemented the scheme in the 


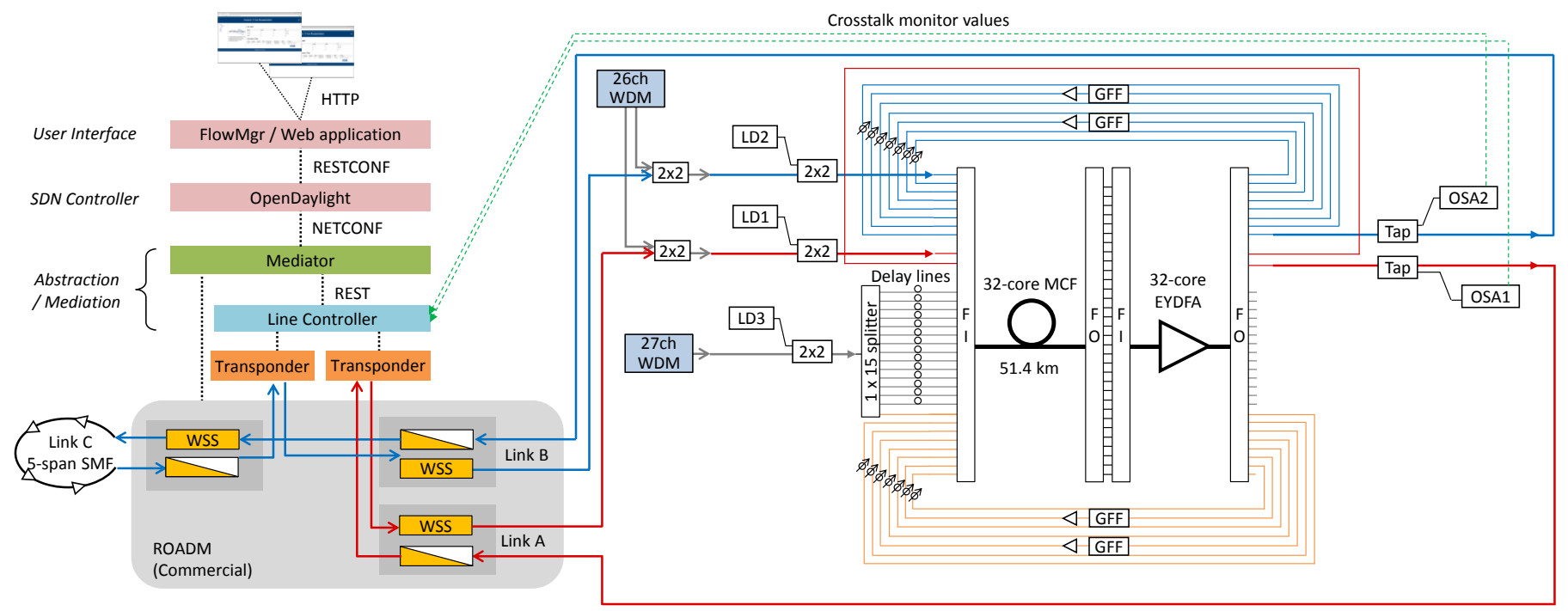

Fig. 5. Experimental setup.

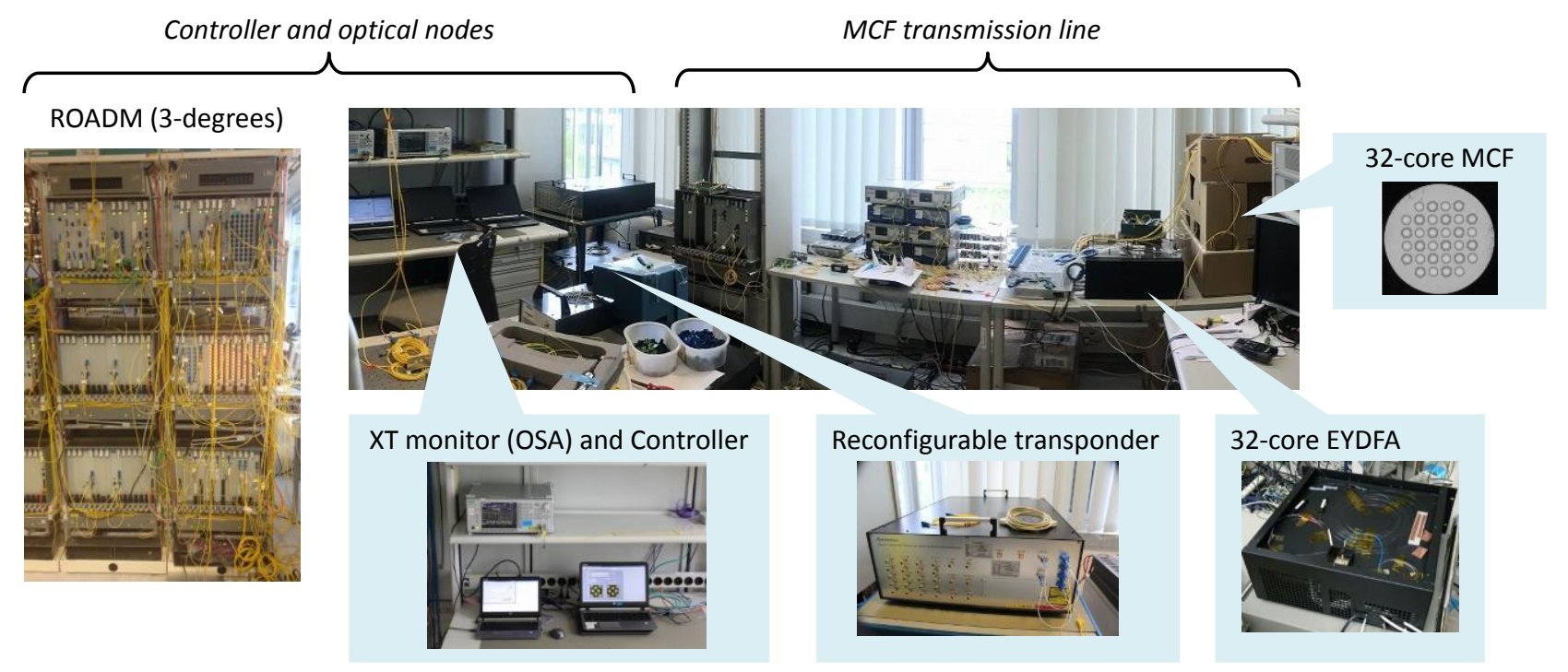

Fig. 6. Photograph of constructed testbed.

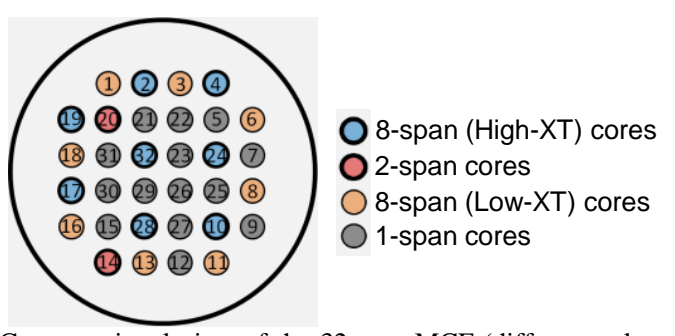

Fig. 7. Cross-sectional view of the 32-core MCF (different colors represent different refractive indices).

MCF links of the testbed.

In the final step, the SDN controller assesses the XT transfer function considering the actual wavelength allocation to determine the optimal modulation format. It configures the transponders and ROADMs to (re)configure the modulation format and the optical path route. The available modulation formats can be acquired by plotting the monitored XT value in Fig. 3, and by selecting the one that has the highest modulation level. In the testbed experiment, we mainly tested and confirmed dynamic operation in the operation phase (Fig. 2).

\section{TestBed SETUP}

Our testbed is as shown in Fig. 5, 6, and 7. State-of-the art optical transport elements were integrated into the testbed to achieve a Pbit/s-class programmable MCF transport network.

\section{A. XT-aware Traffic Engineering Use Case}

The testbed is designed to demonstrate XT-aware traffic engineering, shown in Fig. 8, as a representative use case where "XT-awareness" is important. First, the use case considers a low-priority optical path is established on a low-XT MCF span (Link A in Fig. 8). Since the XT is lower than the required level, the span is capable of using 16QAM modulation format. Next, we assume that a request for high priority optical path arrives that needs to be served over Link A. This forces the route of the low priority path to be changed to a less favorable route (Link $\mathrm{B}-\mathrm{C})$ to make room for the newly-arrived high priority path. Since the XT level of the new route (Link B-C) is higher than 


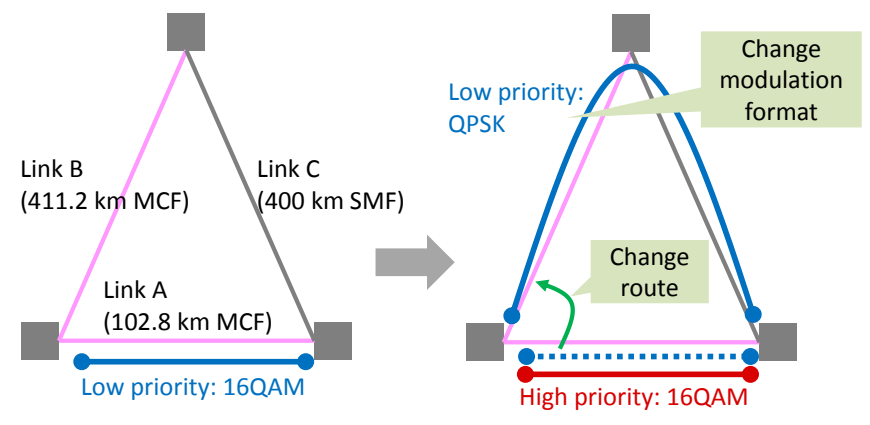

Fig. 8. Use case of XT-aware traffic engineering.

that of the tolerable for 16QAM, the 16QAM format is no longer appropriate and it requires a change towards lower order modulation format, i.e., QPSK or 8QAM.

\section{B. Transmission Line}

The right side of Fig. 5 presents the DSDM transmission line. It consists of a 51.4-km 32-core heterogeneous single-mode MCF [13], a 32-core Erbium-Ytterbium doped fiber amplifier (EYDFA) [9], and in-service inter-core XT monitors [10] (Fig. 4). The MCF has a heterogeneous core arrangement in a square lattice, where two different refractive indices are used to suppress XT. Each core incorporates trench index rings. The fiber is followed by an integrated 32-core EYDFA. The XT and transmission characteristics of this setup were reported in [12].

To model different levels of XT and their effects in an MCF network, sets of cores were connected in series with various combinations. As shown in Fig. 5 and Fig. 7, a set of 8 concatenated cores (cores 2, 4, 10, 17, 19, 24, 28, and 32) surrounding several high input power cores (for XT loading) represents a 400-km high-XT line. Another set of 8 concatenated cores around the outer perimeter of the fiber (cores 1, 3, 6, 8, 11, 13, 16, and 18) represents a 400-km low-XT line. A gain flattening filter (GFF) was inserted after every 3 spans. Further, a set of 2 cores (cores 14 and 20) represents a $100-\mathrm{km}$ line with minimum XT. Such transmission line deployment allows the investigation of different deployment and operation scenarios, including XT-aware traffic engineering, optimization of signal allocation to MCF cores, amplification power saving by concentrating traffic on MCF spans, and so on.

For XT monitoring, two pilot tones are combined with the input WDM signal by a 2 x 2 coupler for each transmission line as shown in Fig. 4, and the output signal is tapped and its spectrum measured by using an optical spectrum analyzer (OSA).

\section{Network Element (NE)}

The testbed employs a commercial ROADM, which provides wavelength switching capabilities that support multi-core use. As such, its colorless-directionless add/drop (CD-A/D) optical filter structure allows it to accept any transponder line signal as alien wavelength clients. These optical client signals are switched and multiplexed to form dense WDM line signals ready for transport over optical links in multiple directions. The network element control interface utilizes Simple Network Management Protocol version 3 (SNMPv3) and operates on a set of standard and private Management Information Base (MIB) modules.

\section{SDN Controller}

The SDN controller in the testbed adopts the hierarchical layer model as shown on the left side of Fig. 5. The controller primarily consists of three parts: an abstraction and mediation part, a SDN controller part, and a user interface part. The abstraction and mediation part is further divided into a Mediator and a Line Controller. This hierarchical layer model intends to ensure not only scalability and flexibility but also interoperability among multiple vendors while remaining future-proof; in the future network equipment is likely to be disaggregated into more finely granular components.

The Line Controller controls and manages the programmable transponders and the MCF transmission line. The Line Controller is connected to one or more transponders and implemented the minimal set of objects required to manage the digital signal processor (DSP) and optical module in the transponder. This implementation enables reconfiguration of the modulation format, wavelength, laser on / off, and so on. In addition, the implementation enables the performance monitoring of transmission-specific parameters such as Q-factor or bit error rate (BER). These configuration and monitoring functions are achieved using a proprietary interface between the Line Controller and the transponders. The controller also collects the monitored XT values at certain intervals from OSAs in the MCF transmission line. For the upper layer, the controller provides the Mediator with an interface that offers transponder configuration and abstracted transmission parameter monitoring functions.

The Mediator integrates the $\mathrm{NE}$ and programmable transponder control interfaces. It communicates with NEs (e.g. ROADM) using SNMP protocol to discover NE configuration and manage the NE cross-connect table. It also communicates with the Line Controller and configures the transponders and accesses the monitoring data as explained above.

As for the SDN controller part, we adopted OpenDaylight [14], which is one of the most acknowledged open-source SDN controllers. The interfaces between OpenDaylight, the Mediator, and the Line Controller are modelled based on the spectral-spatial flow-node data model which is an extension of the subset of objects defined by the OpenDaylight inventory model. OpenDaylight mounts and controls the Mediator as a Network Configuration Protocol (NETCONF) [15] client.

The user interface utilizes common Web application pattern and implements HyperText Markup Language (HTML) server based on the OpenDaylight northbound RESTCONF [16] interface. Thus monitoring the SMF transport network status and configuring optical paths can be realized through a graphical user interface (GUI).

\section{TESTBED RESUlt}

We used the testbed experimental setup shown in Fig. 5 to demonstrate an MCF transport network with in-service XT-aware optical path control [11]. The SDN control layer 


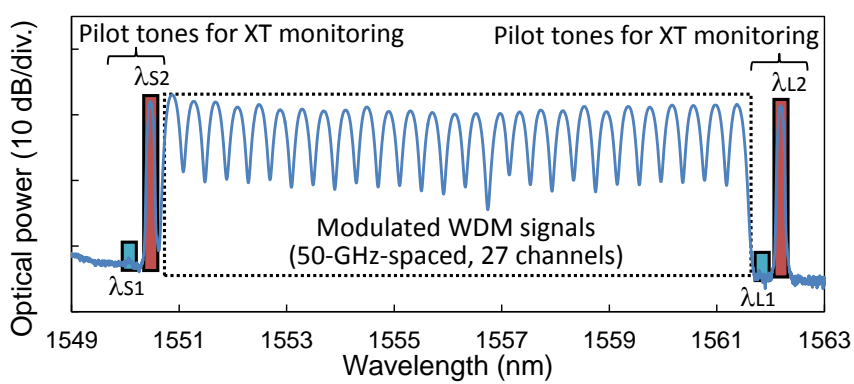

(a)

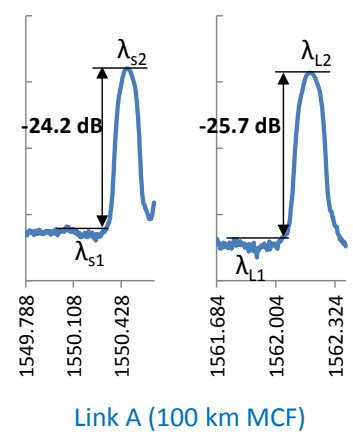

(b)

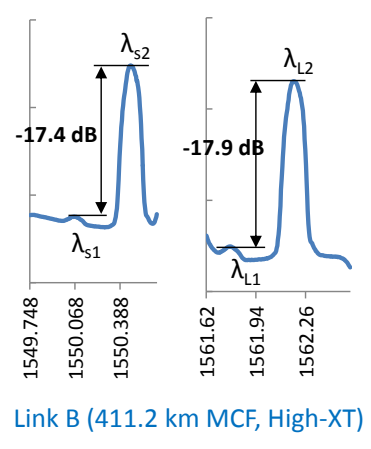

(c)
Fig. 9. Measured XT monitoring performance in the testbed. (a) 27 DWDM channels plus 4 pilot tones in Link A, (b) excerpt of pilot tone channels in Link A, (c) excerpt of pilot tone channels in Link B.

(user interface, SDN controller, and abstraction / mediation function) were used to manage two transponders and a ROADM as well as a collection of transmission-line-specific parameters such as Q-factor, OSNR and XT values via the line controller as described earlier. The test signals at $192.65 \mathrm{GHz}$ $(1556.151 \mathrm{~nm})$ were generated by two programmable transponders capable of switching between $100 \mathrm{Gbit} / \mathrm{s}$ polarization-division-multiplexed (PDM) -QPSK, $150 \mathrm{Gbit} / \mathrm{s}$ PDM-8QAM, and $200 \mathrm{Gbit} / \mathrm{s}$ PDM-16QAM formats. The transponders are connected to the transmission line via a three degree commercial ROADM. One of the directions was a configurable 400-km SMF link ( 5 x $80 \mathrm{~km} / \mathrm{span}$ ). The other two directions were used to switch between different cores or core chains of the MCF.

In the MCF transmission line, the 2-span 100-km line, and the $400-\mathrm{km}$ high-XT line were used as Link A, and B, respectively as shown in Fig. 8. The two test signals, which serve as high and low priority, were combined with different 26 channel $50-\mathrm{GHz}$ spaced WDM signals and injected into the MCF cores forming Link A or B. The remaining cores were used as single-span cores to fill up the transmission line with de-correlated WDM PDM-QPSK signals (27 channels in the range $1550.918-1561.419 \mathrm{~nm}$ ), and to load XT. In each core, two pilot tones are injected together with the WDM signals; $\lambda_{\mathrm{S} 2}=1550.517$ and $\lambda_{\mathrm{L} 2}=1562.233$ for the core under test and $\lambda_{\mathrm{S} 1}=1550.116$ and $\lambda_{\mathrm{L} 1}=1561.826$ for the others.

Figure 9(a) shows the DWDM spectrum measured using the OSA after transmission. The inter-core XT outside both ends of the WDM signal bandwidth were estimated by comparing the optical power differences between the corresponding reference

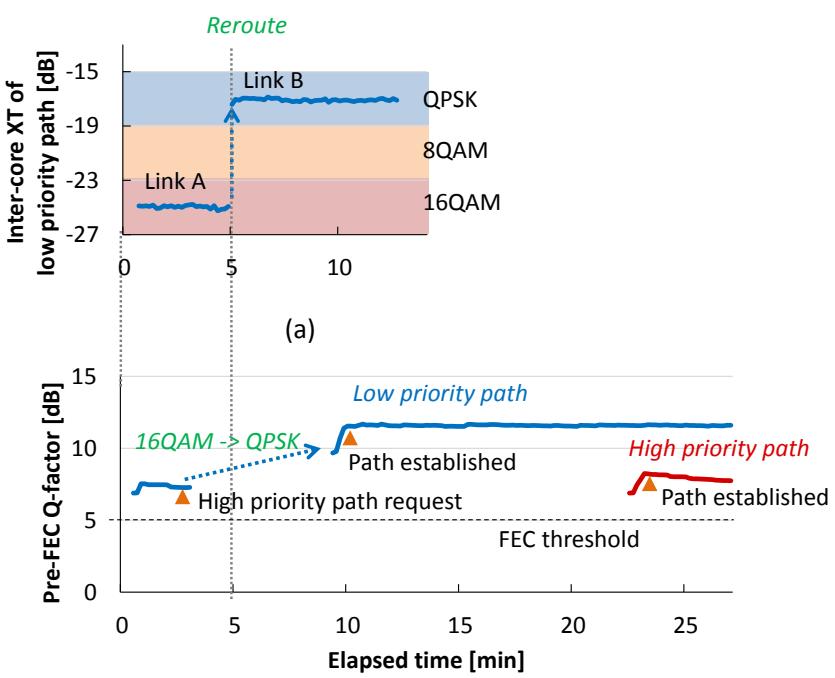

(b)

Fig. 10. Time evolution of (a) inter-core XT of low priority path and (b) Pre-FEC Q-factor.

and XT pilot tones [10], and the XT at the signal wavelength was estimated by linear interpolation of the estimated XT values at the WDM spectra edges. For example, we can see that the XT range of Link A is estimated between $-25.7 \mathrm{~dB}$ and $-24.2 \mathrm{~dB}$ by taking the differences between reference and XT pilot tones at respective WDM spectra edge (the difference between $\lambda_{\mathrm{S} 2}$ and $\lambda_{\mathrm{S} 1}$ at short wavelength, and the difference between $\lambda_{\mathrm{L} 2}$ and $\lambda_{\mathrm{L} 1}$ at long wavelength). By linearly interpolating these values, XT at the wavelength used by the test signal in Link A is estimated to be about $-24.9 \mathrm{~dB}$. Similarly, XT at the same wavelength in Link B is estimated to be about $-17.7 \mathrm{~dB}$. By comparing these estimated XT values with those in Fig. 3, which represent the relationship between Q penalty and XT, we can see that QPSK, 8QAM, and 16QAM formats are available for Link A, while QPSK is the only available modulation format for Link B.

Figure 10 visualizes the real-time measurement data for a low-priority, 16QAM channel initially routed across the 2-core 100-km MCF link (Link A) having low XT. Since the SDN controller continuously collects inter-core XT of the low priority path from OSA via the Line Controller and the Mediator (the value is displayed by the GUI), we can see that the inter-core XT of the low priority path is kept at low (Fig. 10 (a)). When the high priority 16 QAM path demand arrived, the low priority path was pre-empted by the high priority path and rerouted to the route consisting of the 8-core high-XT MCF link and 400-km SMF (Link B-C). Accordingly, the modulation format of the low priority path was changed to QPSK, the only available modulation format (known in advance). After waiting a few minutes for the change of modulation format, the low priority channel was successfully switched to the new route and all the established paths showed stable error free operation after FEC decoding. 


\section{CONCLUSION}

We presented the concept of the single-mode MCF transport network that is orchestrated by SDN controller to address the issue of inter-core XT. In addition, we proposed the SDN-supported XT-aware optical path control scheme using the in-service XT monitoring method. To evaluate the feasibility of the concept and scheme, we constructed an MCF transport network testbed comprising 32-core MCF and EYDFA, programmable transponders, 3-degree commercial ROADM, and hierarchical SDN controller which is capable of collecting XT values in the MCF transmission links by using OSAs. An XT-aware traffic engineering scenario was examined as a use case, and the results confirmed that both appropriate modulation format change based on XT monitoring and optical path routing via ROADMs were achieved dynamically by the SDN controller. In addition, we confirmed that all established paths showed stable error free operation after FEC decoding.

\section{ACKNOWLEDGMENT}

This work was supported by the EU-Japan coordinated R\&D project on "Scalable And Flexible optical Architecture for Reconfigurable Infrastructure (SAFARI)" commissioned by the Ministry of Internal Affairs and Communications (MIC) of Japan and EC Horizon 2020.

\section{REFERENCES}

[1] T. Morioka, "New generation optical infrastructure technologies: "EXAT Initiative" Towards 2020 and beyond," Proc. OptoElectron. Commun. Conf., FT4, 2009.

[2] D. J. Richardson, J. M. Fini, and L. E. Nelson, "Space-division multiplexing in optical fibres," Nature Photonics, Vol. 7, p. 354, 2013.

[3] N. Amaya, M. Irfan, G. Zervas, R. Nejabati, D. Simeonidou, J. Sakaguchi, W. Klaus, B. J. Puttnam, T. Miyazawa, Y. Awaji, N. Wada, and I. Henning, "Fully-Elastic multi-granular network with space/frequency/time switching using multi-core fibres and programmable optical nodes," Opt. Express, vol. 21, no. 7, pp. 8865-8872, 2013.

[4] V. A. J. M. Sleiffer, H. Chen, Y. Jung, P. Leoni, M. Kuschnerov, A. Simperler, H. Fabian, H. Schuh, F. Kub, D. J. Richardson, S. U. Alam, L. Grüner-Nielsen, Y. Sun, A. M. J. Koonen, and H. de Waardt, "Field demonstration of mode-division multiplexing upgrade scenarios on commercial networks," Opt. Express, vol. 21, no. 25, p. 31036, 2013.

[5] G. M. Saridis, B. J. Puttnam, R. S. Luis, W. Klaus, T. Miyazawa, Y. Awaji, G. Zervas, D. Simeonidou, and N. Wada, "Experimental demonstration of a flexible filterless and bidirectional SDM optical metro/inter-DC network," Proc. Eur. Conf. Opt. Commun., M.1.F.3, 2016.

[6] S. Fujii, Y. Hirota, H. Tode, and K. Murakami., "On-demand spectrum and core allocation for reducing crosstalk in multicore fibers in elastic optical networks," J. Opt. Commun. Netw., Vol. 6, no. 12, p. 1059, 2014.

[7] T. Kobayashi, M. Nakamura, F. Hamaoka, K. Shibahara, T. Mizuno, A. Sano, H. Kawakami, A. Isoda, M. Nagatani, H. Yamazaki, Y. Miyamoto, Y. Amma, Y. Sasaki, K. Takenaga, K. Aikawa, K. Saitoh, Y. Jung, D. J. Richardson, K. Pulverer, M. Bohn, M. Nooruzzaman, and T. Morioka, "1-Pb/s (32 SDM/46 WDM/768 Gb/s) C-band dense SDM transmission over 205.6-km of single-mode heterogeneous multi-core fiber using 96-Gbaud PDM-16QAM channels," Proc. Eur. Conf. Opt. Commun., PDP Th5B.1, 2017.

[8] T. Mizuno, K. Shibahara, F. Ye, Y. Sasaki, Y. Amma, K. Takenaga, Y. Jung, K. Pulverer, K. Saitoh, S. Matsuo, K. Aikawa, M. Bohn, D. J.
Richardson, Y. Miyamoto, and T. Morioka, "Long-haul dense space-division multiplexed transmission over low-crosstalk heterogeneous 32-core transmission line using a partial recirculating loop system," J. Lightw. Technol., vol. 35, no. 3, pp. 488-498, 2017.

[9] S. Jain, T. Mizuno, Y. Jung, A. Isoda, K. Shibahara, J. R. Hayes, Y. Sasaki, K. Takenaga, Y. Miyamoto, S. Alam, and D. J. Richardson, "Improved cladding-pumped 32-core multicore fiber amplifier," Proc. Eur. Conf. Opt. Commun., Th.2.D.2, 2017.

[10] T. Mizuno, A. Isoda, K. Shibahara, Y. Miyamoto, S. Jain, S. U. Alam, D. J. Richardson, C. Castro, K. Pulverer, Y. Sasaki, Y. Amma, K. Takenaga, K. Aikawa, and T. Morioka, "In-service crosstalk monitoring for dense space division multiplexed multi-core fiber transmission systems," Proc. Opt. Fiber. Commun. Conf. Exhib., M3J.2, 2017.

[11] K. Pulverer, T. Tanaka, U. Häbel, C. Castro, M. Bohn, T. Mizuno, A. Isoda, K. Shibahara, T. Inui, Y. Miyamoto, Y. Sasaki, Y. Amma, K. Aikawa, S. Jain, S. Alam, D. J. Richardson, M. Nooruzzaman, and T. Morioka, "First demonstration of single-mode MCF transport network with crosstalk-aware in-service optical channel control," Proc. Eur. Conf. Opt. Commun, Th.PDP.B.5, 2017.

[12] C. Castro, S. Jain, Y. Jung, E. De Man, S. Calabrò, K. Pulverer, M. Bohn, J. Hayes, S. Alam, D. J. Richardson, Y. Sasaki, T. Mizuno, K. Shibahara, T. Kobayashi, Y. Miyamoto, T. Morioka, and W. Rosenkranz, "Crosstalk analysis of 32-core dense space division multiplexed system for higher order modulation formats using an integrated cladding-pumped amplifier," Proc. Eur. Conf. Opt. Commun, M.2.E.5, 2017.

[13] Y. Sasaki, R. Fukumoto, K. Takenaga, K. Aikawa, K. Saitoh, T. Morioka, and Y. Miyamoto, "Crosstalk-managed heterogeneous single-mode 32-core fibre," Proc. Eur. Conf. Opt. Commun, W.2.B.2, 2016.

[14] OpenDaylight community, web-site, http://www.opendaylight.org/

[15] Network Configuration Protocol, IETF RFC 6241.

[16] RESTCONF Protocol, IETF RFC 8040. 